tion. The index case was a 43-year-old woman in whom acute icteric hepatitis B developed approximately 4 months after treatment at an autohemotherapy clinic in October and November 1997. Following the initiation of case-finding procedures, additional hepatitis B surface antigen (HBsAg)-positive cases were identified, as well as one case of hepatitis $\mathrm{C}$ virus $(\mathrm{HCV})$ seroconversion.

Both $\mathrm{HBV}$ and $\mathrm{HCV}$ serological testing were performed on serum samples from identified patients and clinic staff. Patients provided the dates that they received autohemotherapy by completing a questionnaire. Serum samples from 352 of the 399 patients were tested for markers of $\mathrm{HBV}$ and $\mathrm{HCV}$ infection. Among the 352 patients, 54 (15\%) had evidence of HBV infection, 23 had markers of resolved infection, and 31 had evidence of active HBV infection on initial testing. Among the patients who had evidence of resolved $\mathrm{HBV}$ infection, 6 had a recently resolved infection. HBV DNA could be amplified and sequenced from 32 of the $33 \mathrm{HBsAg}$-positive patients and staff members. Complete nucleotide identity in both the surface and core genes was demonstrated in 30 of the 32 cases sequenced; the outbreak-associated HBV belonged to genotype $\mathrm{D}$. The proportion of patients with markers of $\mathrm{HBV}$ infection increased with the number of treatments received (chi-square=18, $P<.0001$ ). The attack rate also was associated with the week of visit $(P=.011)$.

Five of the 352 patients had positive test results for antibodies against HCV, including the index case. The only known risk factor for $\mathrm{HCV}$ infection in the index case was autohemotherapy. The 4 other patients had chronic HCV infection before they attended the clinic. However, HCV genotype testing results were unique for each of the 5 patients. Antibodies against hepatitis delta virus were negative in all patients.

Fifty household contacts and sexual partners of patients exposed to HBV also were tested; 24 contacts of patients with active HBV infection were vaccinated. Acute icteric hepatitis developed in 1 sexual contact; analysis revealed that she and her partner had HBV strains of the same genotype.

Four family members, one of whom was a registered physician, staffed the clinic. Acute HBV developed in two of the four in March 1998; one tested positive for antibodies against $\mathrm{HCV}$ but negative for HCV RNA. Results in the third showed resolved $\mathrm{HBV}$ and tested positive for antibodies against HCV but negative for HCV RNA. The fourth had no markers for $\mathrm{HBV}$ or $\mathrm{HCV}$ infection. The four people involved had performed autohemotherapy on each other during the previous year.
FROM: Webster GJM, Hallet R, Whalley SA, Meltzer M, Balogun K, Brown D, et al. Molecular epidemiology of a large outbreak of hepatitis B linked to autohaemotherapy. Lancet 2000;356:379-384.

\section{Hematological Changes in Workers From Ethylene Oxide Exposure}

Shaham and colleagues from the Occupational Cancer Department, National Institute of Occupational and Environmental Health, Tel-Aviv University, Raanana, Israel, conducted a cross-sectional study to determine whether occupational exposure to low levels of ethylene oxide can cause hematological abnormalities. Blood samples were collected from a group of 47 hospital workers who were exposed to ethylene oxide during a mean period of 6.6 years (standard error, 1.1). Ethylene oxide range levels measured were $<0.01$ to $0.06 \mathrm{ppm}$. The control group, individually matched by age, gender, and smoking habits, consisted of 88 workers from the administrative sector.

They found significant differences between the exposed and control groups in the frequency of workers with white blood cells lower than the normal range. Although there was no significant difference in the absolute mean number of the total white blood cells, they found an elevation in the absolute mean number of monocytes and eosinophils $(P<.01)$ and a decrease $(P<.01)$ in the absolute mean number of lymphocytes in the exposed group compared with the control group. They also found an elevation $(P<.01)$ in the percentage of hematocrit and the mean absolute number of the red blood cells, and a decrease $(P<.01)$ in the mean absolute number of platelets in the exposed group compared with the control group. The mean absolute number of eosinophils, red blood cells, and percentage of hematocrit was significantly higher, and the mean absolute number of lymphocytes and platelets was significantly lower in the subgroups with a higher cumulative dose of exposure. A positive dose-response was found between cumulative dose exposure and the absolute mean number of eosinophils.

The authors suggest that the use of complete blood cells with differential in routine medical surveillance and for early detection of hygiene problems should be reexamined with special attention to the eosinophils count.

FROM: Shaham J, Levi Z, Gurvich R, Shain R, Ribak J. Hematological changes in hospital workers due to chronic exposure to low levels of ethylene oxide. J Occup Environ Med 2000;42:843-850. 